\title{
EVALUATING PUBLIC TRANSPORT PERFORMANCES BY UTILIZING PROBE VEHICLES
}

\author{
Marko Matulin ${ }^{1}$, Štefica Mrvelj ${ }^{2}$, Niko Jelušić ${ }^{3}$ \\ ${ }^{1,2,3}$ Faculty of Transport and Traffic Sciences, University of Zagreb, Vukelićeva 4, 10000 Zagreb, Croatia
}

Received 8 November 2013; accepted 22 April 2014

\begin{abstract}
The work presented in this paper originates from the research conducted within the CIVITAS ELAN project, co-funded by the European Union. One of the main project objectives was to improve the quality of public transport (PT) service in the city of Zagreb. For the purpose of evaluating the impact of the project, it was necessary to analyze and evaluate the tramway network performances with high level of detail. Owing to this requirement, four probe vehicles (trams) and GPS (Global Positioning System) vehicle tracking technique was used to collect the traffic data which enabled accurate calculation of operational performances of the network. The selected technique made the evaluation possible on micro and macro level. Apart from presenting the evaluation results, the paper outlines several case specific disadvantages of GPS probe vehicle technique. The two main ones were a mismatch between geographical locations of the control points and actual tram position recorded by GPS receiver and inability to distinguish dwell time and intersection delay if the PT stop is located directly in front of signalized intersection. This knowledge can be useful for future research endeavors in this field.
\end{abstract}

Keywords: network performances, public transport, probe vehicle, GPS, data collection, evaluation.

\section{Introduction}

In the wide spectrum of public services offered to the citizens of major cities, public transport (PT) service can certainly be considered as one of the most important. To defend this statement it can be mentioned that, in the European context, the PT system is often described as 'the backbone of the cities'. Nevertheless, the advent of two main challenges which PT operators are facing in recent years can be observed: a) they are continuously struggling with the need to reduce operational costs and $b$ ) they have to keep up with the increasing passenger' requirements in terms of the quality of delivered service. It is clear that these two challenges are somewhat in collision with each other. Firstly, PT operators are dealing with the reduction of public funding, increasing energy prices and competition on the transportation market. Secondly, passengers demand fast and reliable services which have to be provided in the state-of-the art PT vehicles.

During the implementation of the large scale, collaborative CIVITAS ELAN project (CIVITAS Initiative, 2012), which occurred in the 4.5 year period in the city of Zagreb (from 2008 to 2013), the PT service was considerably improved. Specifically, new PT vehicles were introduced with better operational characteristics, safety

${ }^{1}$ Corresponding author: mmatulin@fpz.hr

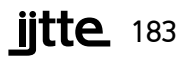


and security conditions were improved and new PT priority system for trams was demonstrated. These measures had to ensure that PT stayed one of the main transport modes in the city and that PT service becomes fast and reliable which would be highly beneficial for all subjects on the transportation market (the city municipality, PT operator and service users).

From the traffic engineering point of view, the last abovementioned project activity (demonstration of the PT priority system which can alter signal plans, ensuring the "green" period for trams when they approach intersections) was especially interesting, because it imposed the need for detailed analysis and evaluation of tram network performances. Most of the project measures were implemented in a predefined demonstration area located near the city centre. This, so called, corridor only partially covers the total length of specific tram lines. In turn, this meant that performance improvements, achieved with the demonstration of the PT priority system, would occur only on specific segment of the PT network, while there would be no real changes outside of the corridor. Additionally, due to technical issues which arose and remained during the project lifetime, it was decided to demonstrate the priority system on one intersection for a limited time period. Therefore, it was paramount to investigate the network performances with relatively high level of detail in order to enable the evaluation on intersection-per-intersection basis. This required very detailed set of data which describe the performances of PT network.

In the beginning of our research the first question which arose was: what where the common indicators of tram network performances?
While reviewing the literature on this matter we found that the performances are often ill-defined or that different authors make different interpretations of what the performances are and how they are measured. In general, the performances are often expressed by a mix of quantitative and qualitative indicators. The boundary between PT performance and PT quality of service has proven to be vague as well.

This lack of standardization was already indicated by Pullen who argued for improved definition and clarification (Pullen, 1991). In Harrison et al. (1998), authors defined "hard" quality indicators as those which are more quantifiable (e.g. access time) and "soft" quality indicators as "non-journey time attributes" such as information provision, staff attitude and satisfaction. Prioni and Hensher (2000) grouped bus performance indicators into six quality dimensions, also deploying the concept of "hard" and "soft" indicators.

In 2002, EU deployed a standard for certification of $\mathrm{PT}$ system performances (EN13816) which categorizes performance indicators (qualitative and quantitative) into 8 categories: availability (network, operation time, reliability), access (interfaces, ticketing), information (travel information), time (travel time, punctuality, regularity), customer service (availability of personal, competence, assistance), comfort (space, driving), safety (criminal attacks, accidents) and environment, (European standards, 2013). In Egmond et al. (2003) four levels of PT performances are defined: external, strategic, tactical and operational. Different levels are focusing on population attributes, population density, political interest and regulations, organizational and financial framework analysis, accessibility of different 
PT modes, intermodality, marketing and information. Some authors devote higher importance to the user perspective of PT performance and argue that "hardcore" performances are good indicator for service provider, but "true" performance can only be evaluated with customer satisfaction survey (e.g. Thompson and Schofield, 2007).

Apart from these differences in understanding of PT network performances, the quantitative indicators are sometimes only represented by the monetized values of network delay, operation time and/or other measureable parameters, meaning that the performances are evaluated from the perspective of economists. That kind of analyses do not necessarily give an insight into full impact of different measures (e.g. this is the case in Currie et al., 2005; Currie et al., 2007; Vedagiri and Arasan, 2009).

For the purpose of evaluating different impacts of the PT priority system, we were focused exclusively on the operational performances of specific tram lines which traverse through the corridor. The lack of clarity of PT network performance definitions encouraged us to define our own evaluation indicators which we then used for the evaluation of the priority system in Zagreb. The defined indicators required detailed data sets so that evaluation of operational performances would be possible per specific segment of the lines. The ability to record tram position and speed with relatively high resolution of the measurement (e.g. each second) encouraged us to use probe vehicles (four trams) and GPS (Global Positioning System) tracking technique. The measurements lasted over the period of two weeks before and two weeks after the implementation of the system. This paper brings the results of this analysis and points out few case specific disadvantages of this data collection methodology.

The remainder of the paper is structured as follows. Section 2 describes our performance indicators used in evaluation. Brief description of data collection methodology can be found in Section 3. Section 4 brings evaluation results and discussion, while Section 5 concludes.

\section{Performance Indicators}

\subsection{Description}

As it is evident from the previous chapter, the focus is on tram network performances. Although obvious, it is useful to describe one journey of a tram before presenting the performance indicators. The journey has two termination points (origin terminal $\mathrm{A}$ and destination terminal B) and a finite number of PT stops and signalized intersections in between (illustrated on Fig. 1). It is quite clear that the main impact of the PT priority system on the operational performances of trams is the reduction of intersection delay. This reduction should result in a decrease of running times between adjacent PT stops and terminals of the same PT line.

Nevertheless, sometimes there is no reduction due to the various background impacts that are caused by: a) mixed traffic conditions, b) variability of the number of PT users and the number of trams in operation, c) variable time-gaps between $P T$ vehicles and $d$ ) partial implementation of PT priority system (Matulin et al., 2010). For instance, due to mixed traffic conditions, in the peak periods of the day, when transport demand is at its highest levels, queues of cars can be formed in front of signalized intersections, and block the tram tracks. 
The tram pathway can also be blocked by traffic accidents which results in further performance deterioration. In this case, positive impacts of giving priority to trams at signalized intersections can be easily cancelled out, because trams cannot reach the intersections (Matulin et al., 2011). Sun et al. (2008) detected and described these complex interactions between $\mathrm{PT}$ vehicles and general traffic vehicles in such mixed traffic environments.

In addition, possible increase of PT users could require more trams in order to satisfy the increased transport demand. More trams in operation increases the congestion, i.e. the time-gap between two trams, which traverse through the same network segment in the same direction, can become too small. This increases the possibility that several trams arrive at the same PT stop at the same time, which, in turn, increases dwell times. Increased dwell times may increase operation time and passenger travel times, even though trams might get the priority at intersections.

Effects of the PT priority system could also be reduced if the system itself is partially implemented. This can happen if:

1. The system is not implemented on all signalized intersections of the selected line/corridor (in this case trams could pass through one intersection and cause a blockage on the consecutive intersection).

2. The priority equipment is not installed in all trams which travel on the same line/corridor (in this case a tram which is not equipped with the priority equipment could disturb traffic flow of trams which are equipped).

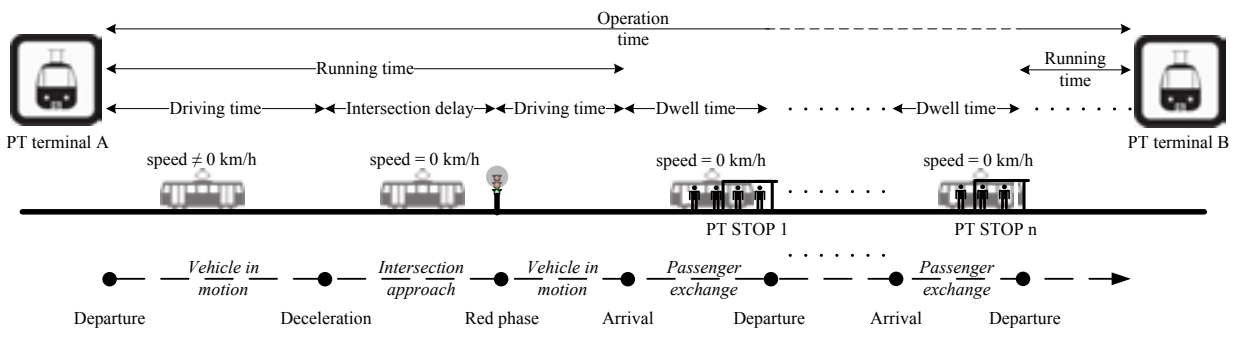

Fig. 1.

Operation Time Decomposition

All of the above described background impacts affect the operational performance of the network. During the analysis of the possible benefits of a PT priority system, such impacts must not be ignored. In our case, when the system is implemented only on one part of the tram line, certain improvements which are achieved on a micro level (e.g. on specific intersection or between two adjacent PT stops) could remain undetected if operational performance of each line segment is not evaluated. Therefore, we decomposed tram operation time into smaller time segments and defined evaluation indicators (Fig. 1).

Since we conducted the measurements in predefined demonstration area of the project (i.e. the corridor), by the operation time we consider the time that elapses from the 
entering of a tram into the corridor to the exit from the corridor.

\subsection{Mathematical Formulation}

In this chapter we provide the mathematical formulation of evaluation indicators which are depicted on Fig. 1. The total tram operation time $\left(T_{o p}\right)$ is given by Eq. (1):

$T_{o p}=T_{d w}+T_{r t}$

where $T_{d w}$ is the total time spent at PT stops and $T_{r t}$ is the total time spent on running between PT stops by the tram on a journey between two terminals. For a known length of a PT line $\left(S_{P T \text { line }}\right)$ and after calculating $T_{o p}$

, operating speed of PT vehicle $\left(v_{o p}\right)$ can be derived (Eq. (2)):

$v_{o p}=\frac{S_{P T l i n e}}{T_{o p}}$

Thus, $T_{o p}$ enables evaluation of PT performances on macro level. The total time spent at PT stops is a sum of dwell times elapsed at different PT stops of the same PT line (Eq. (3)):

$T_{d w}=\sum_{i=1}^{n} t_{d w_{i}}$

where $t_{d w_{i}}$ is the dwell time for PT stop $i$ and $n$ is the number of PT stops on PT line. The total running time between PT stops is equal to Eq. (4):

$T_{r t}=\sum_{j=1}^{n-1} t_{r t_{j, j+1}}$ where $t_{r t_{j, j+1}}$ is the running time between two adjacent $\mathrm{PT}$ stops and $n$ is the number of PT stops. The first PT stop is the origin terminal A and $n$-th PT stop is the destination terminal $\mathrm{B}$. The running time between two adjacent PT stops $\left(t_{r t_{j, j+1}}\right)$ is now decomposed into Eq. (5):

$t_{r t_{j, j+1}}=t_{d t_{j, j+1}}+\sum_{i=1}^{m} t_{i d_{i}}+\sum_{k} t_{l o s s_{k}}$

where $t_{d t_{j, j+1}}$ is the time which PT vehicle spends in motion between two adjacent PT stops (i.e. driving time) and $\sum_{i=1}^{m} t_{i d_{i}}$ is the total intersection delay which is caused at finite number of intersections $m$ between two adjacent PT stops $(j, j+1)$. Any additional loss of time (e.g. delay caused by mixed traffic conditions) is included in $\sum_{k} t_{\text {loss }_{k}}\left(k=0, \ldots, N_{\text {where }} N\right.$ is the number of events which caused unplanned vehicle stopping). Note that expression $\sum_{i=1}^{m} t_{i d_{i}}$ can can be equal to 0 in two cases; firstly, when $\mathrm{PT}$ vehicle passes through the intersection without stopping, and secondly, when there are no intersections between two adjacent PT stops.

The speed per segment $\left(v_{j, j+1}\right)$ is the distance travelled between two adjacent PT stops $\left(s_{j, j+1}\right)$ divided by $t_{r t_{j, j+1}}$ (Eq. (6)):

$v_{j, j+1}=\frac{s_{j, j+1}}{t_{r t_{j, j+1}}}$

Thisset ofindicators $\left(t_{d w_{i}}, t_{r t_{j, j+1}}, t_{d t_{j, j+1}}, t_{i d_{i}}\right.$ and $v_{j, j+1}$ ) enables micro level evaluation of $\mathrm{PT}$ performances. 


\section{Data Collection}

\subsection{Method Description}

In order to collect the data about the operation time $\left(T_{o p}\right)$ and its segments we decided to use four trams as probe vehicles and GPS vehicle tracking technique. The GPS receivers were installed in four trams travelling on the same line. The time-gap between the trams varied, but it remained relatively large throughout the measurements (over one hour at least). The measurements were conducted twice: before and after implementation of the PT priority system. Recordings took place in two week period (Monday to Sunday), each day from 6 a.m. to 10 p.m. Every GPS receiver recorded the tram position and actual speed each second. Recorded GPS data points were extracted from the devices and imported into computer for the analysis. Setting up the recording interval to one second provided us with the high resolution of the measurement which was essential for the network performance evaluation by the defined indicators. Nevertheless, apart from good measurement resolution which was highly appreciated feature, this method also created case specific issues which had to be resolved in the process of data analysis.

\subsection{Case Specific Problems}

The abovementioned issues were manifested as a mismatch between geographical locations of the control points (PT stops or signalized intersections) and actual tram position recorded by the GPS receiver. The GPS vehicle tracking method gives very accurate results for $T_{o p}$ and $v_{o p}$. However, when the tram actual speed is around $0 \mathrm{~km} / \mathrm{h}$, due to the GPS signal reflection, GPS tracks can be in offset to about 30-40 meters. Without map matching it is impossible to determine actual tram position in a specific moment of time, which is important for calculation of different operation time segments.

In case when two trams arrive on the same PT stop in the same time, as it is depicted in Fig. 2, with the GPS vehicle tracking method it is not possible to determine the exact reason why tram B stopped. In this case specific situation, geographical location of the PT stop and the position of $\operatorname{tram~B}$, when the speed is $0 \mathrm{~km} / \mathrm{h}$, do not overlap. Knowing the GPS signal reflection problems, during the data processing it is hard to determine whether the tram B has reached the PT stop and started to alight and board passengers or another vehicle (tram or even individual vehicle) was occupying the stop at the time.

$$
\text { Speed of a tram } B=0 \mathrm{~km} / \mathrm{h} \quad \text { Speed of a tram } A=0 \mathrm{~km} / \mathrm{h}
$$

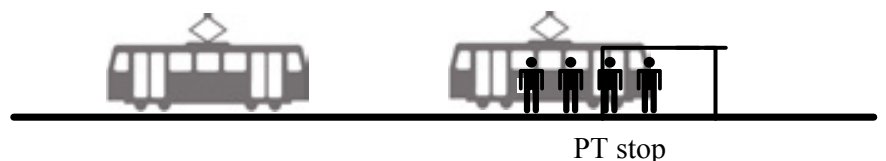

Fig. 2.

Arrival of Two Trams on the Same PT Stop 
It is also noteworthy to mention that when the PT stop is located directly in front of signalized intersection and the "red" period is activated (Fig. 3), intersection delay $\left(t_{i d_{i}}\right)$ and dwell time $\left(t_{d w_{i}}\right)$ measurements are incomplete. This results in inaccurate calculation of driving time $\left(t_{d t_{j, j+1}}\right)$ and speed per segment $\left(v_{j, j+1}\right)$. While processing the GPS data it can be easily detected when the tram speed was $0 \mathrm{~km} / \mathrm{h}$, but in this case the difference between $t_{d w_{i}}$ and $t_{i d_{i}}$ cannot be recognized.

Speed of a tram $=0 \mathrm{~km} / \mathrm{h}$

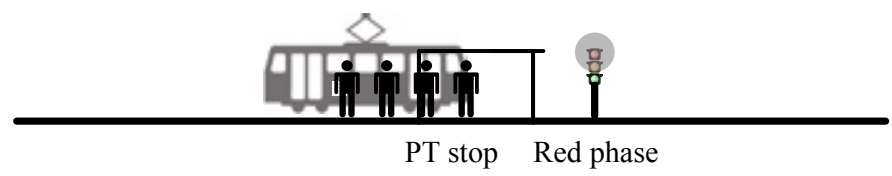

Fig. 3.

Specific Geographical Location of PT Stops

In order to complete the GPS data sets and to cope with the abovementioned issues, we conducted manual measurements of $t_{d w_{i}}$ for those PT stops which are located in front of signalized intersections. Lastly, when a tram B was behind of a tram A (Fig. 2) we used $t_{\text {loss }_{k}}$ variable (Eq. (5)) to quantify the time during which the speed of a tram $B$ was equal to $0 \mathrm{~km} / \mathrm{h}$.

\section{Evaluation of the PT Priority System}

In this section we present the results of the data analysis, i.e. the tram network performances in the corridor before and after the demonstration of the PT priority system (only for one direction of travel).
Note that the priority system was installed on Deželićeva intersection which is located between Trg_marš_tita stop and Frankopanska stop.

According to the Eq. (1), operation time $\left(T_{o p}\right)$ is calculated by adding up tram running time and dwell time. Operating speed $\left(v_{o p}\right)$ is then derived by dividing the length of a tram line that traverses through the corridor $\mathbf{2} .855$ $\mathrm{m})$ and $T_{o p}$. Results of this analysis can be found in Table 1. The following figures (Figs. 4-6) depict average running time between two consecutive PT stops $\left(t_{r t_{j, j+1}}\right)$, average intersection delay for each intersection in the corridor $\left(t_{i d_{i}}\right)$ and average dwell time for each PT stop in the corridor $\left(t_{d w_{i}}\right)$, respectively.

Table 1

Difference between the Average $T_{o p}$ and $v_{o p}$

\begin{tabular}{|l|l|l|l|}
\hline Indicator & Before implementation & After implementation & Difference $=$ After - Before \\
\hline Average $T_{o p}$ & $00: 14: 58$ & $00: 14: 00$ & -58 seconds, i.e. $-6.46 \%$ \\
\hline Average $v_{o p}$ & $11.45 \mathrm{~km} / \mathrm{h}$ & $12.24 \mathrm{~km} / \mathrm{h}$ & $+0.79 \mathrm{~km} / \mathrm{h}$, i.e. $+6.9 \%$ \\
\hline
\end{tabular}

The data from the Table 1 shows that the average $T_{o p}$ was decreased by 58 seconds or $6.46 \%$, while the average $v_{o p}$ is increased by $6.9 \%$. This positive impact is the result of the reduction of average running times between PT stops, which is indicated in 
Fig. 4. Average running time in the whole corridor is reduced by $7.3 \%$ (this is the difference between the sum of all running times before and after the implementation of the system). More detailed analysis of running time shows that the average running time, on the part of the line where the priority is introduced (between Trg_marš tita and Frankopanska stop), was reduced by significant 23 seconds.

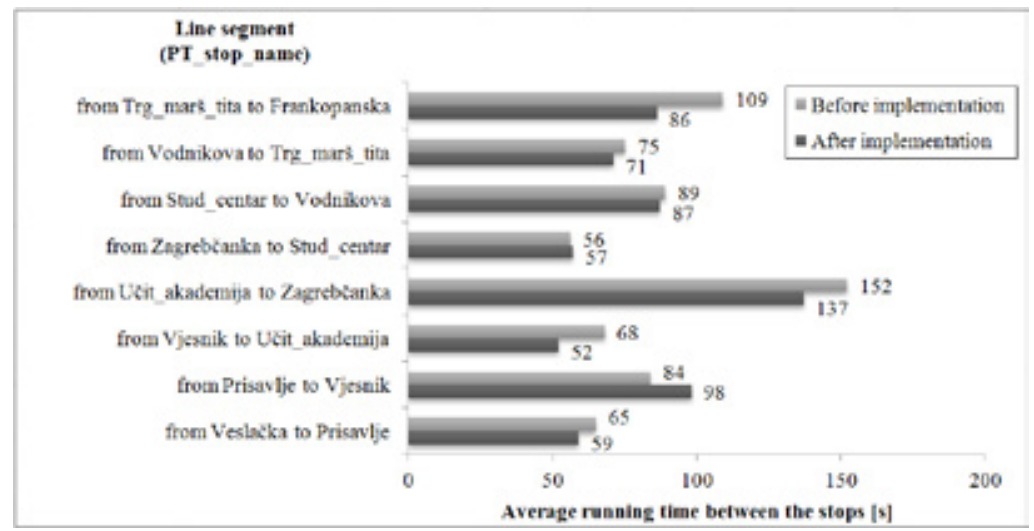

Fig. 4.

Average Running Times between Two Consecutive PT Stops

The largest savings in tram operational time, i.e. running time, were achieved on the part of the corridor where the PT priority system was demonstrated (Deželićeva intersection). The average delay on that intersection alone is reduced by 21 seconds (depicted on Fig. 5). Cumulative intersection delay in the corridor was reduced by 43 seconds or $17.84 \%$. Notwithstanding, that reduction is not entirely due to the priority system, because car traffic flow analysis showed that, during the second round of data collection (i.e. after the implementation), there was a decrease in the number of cars in the corridor. The reduction is most likely caused by the impact of the global economic crisis and the recession which followed. This reasoning only accentuates the need for detail performance evaluation which often has to be complemented by background measurements to be able to determine the origin of changes in real-life traffic systems.
Analysis of dwell time showed that cumulative dwell time was reduced by 7 seconds which also contributed to the reduction of average running time in the corridor. Detailed analysis shows that dwell times remained almost the same on most PT stops ( \pm 4 or 5 seconds). However, as depicted on Fig. 6, we see considerable decrease of the average dwell time on the Stud_centar PT stop. This decrease can be explained by the fact that during the second round of data collection new trams were in operation. Introduction of the new trams was also one of the objectives of the CIVITAS ELAN project. New trams are low floored meaning that senior users and users with different physical impairments can board/alight trams easier. Prior to the introduction of new trams this particular PT stop imposed a problem for these groups of users, because the curb stone at that specific stop was 30 to $40 \mathrm{~cm}$ lower than the doorstep 
of the older type of trams and that made older trams difficult to board/alight. We consider this as an excellent example that shows how detailed analysis of network performances can give important insights into impacts which different measures are producing. Nevertheless, these impacts could have remained undetected without detailed data sets or, in our case, without hundreds of thousands of recorded GPS data points.

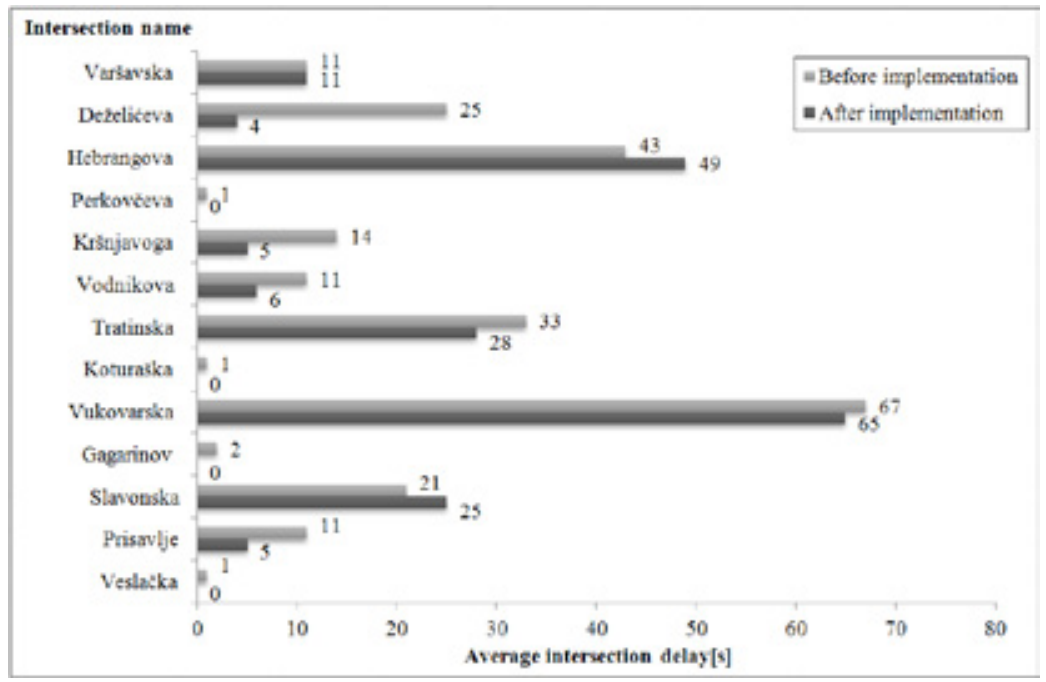

Fig. 5.

Average Intersection Delay in the Corridor

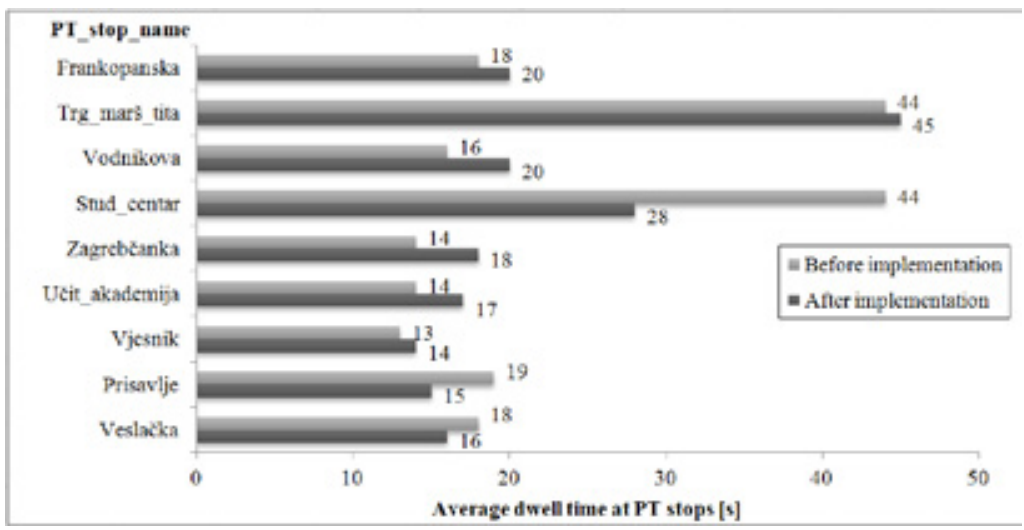

Fig. 6.

Average Dwell Time at PT Stops in the Corridor

As it can be seen from the presented results, GPS vehicle tracking provided us with enough data to be able to conduct very detailed evaluation of the network performances. The collected data made evaluation possible on a micro level (i.e. 
the evaluation of the system per specific intersection or PT stop) and on the macro level (i.e. the evaluation of network performances for the whole corridor).

\section{Conclusion}

The introduction of the PT priority system imposed different research requirements in all phases of its implementation and evaluation. In this paper we were focused only on the last phase, i.e. evaluation, for which we defined several performance indicators. Due to specific implementation conditions and restraints (various background impacts and partial implementation of the system) it was recognized that only full data sets will suffice if we want to be able to conduct detailed performance evaluation of the tram network. The ability to record tram speed and position each second was considered as important requirement which data collection method had to fulfil, thus probe vehicles and GPS tracking technique was selected.

As it can be seen from the presented results, the selected data collection method made evaluation possible on micro and macro level, i.e. per specific intersection and PT stop, or on the whole corridor. This ability was crucial for the project evaluation and we believe that this knowledge can be highly beneficial for future investigations of PT network performances. This is especially the case for those projects which bring the improvements only on certain network segments and not on the whole network. The same approach can be easily applied in other networks (e.g. bus networks) which makes it highly adaptable.

Nevertheless, we were also able to identify few drawbacks of this methodology. The two main ones were: a) mismatch between geographical locations of the control points (PT stops and intersections) and actual tram position recorded by GPS receiver which requires additional attention in the data analysis process and b) inability to distinguish dwell time and intersection delay if the PT stop is located directly in front of signalized intersection.

Based on these findings we can conclude that probe vehicles, together GPS vehicle tracking technique, provide enough data for very detailed evaluation, however, it is wise to supplement it with other methods (e.g. onsight measuring, video image analysis, etc.) in order to avoid abovementioned drawbacks.

\section{Acknowledgments}

This paper is inspired by the research undertaken in the CIVITAS ELAN project (Grant Agreement No.: ELAN TREN/ FP7TR/218954/"ELAN"). We wish to thank all project partners, especially Zagreb's public transport company (ZET) for enabling us to conduct the measurements. 


\section{References}

CIVITAS Initiative. 2012. Available from Internet: <http://www.civitas.eu/content/elan>.

Currie, G.; Sarvi, M.; Young, B. 2005. Road space allocation for public transport priority. In Proceedings of the AITPM National Conference.

Currie, G.; Sarvi, M.; Young, B. 2007. A new approach to evaluating on-road public transport priority projects: balancing the demand for limited road space, Transportation. DOI: http://dx.doi.org/10.1007/s11116006-9107-3, 34(4): 413-428.

Egmond, P.; Nijkamp, P.; Vindigni, G. 2003. A comparative analysis of the performance of urban public transport systems in Europe. Oxford: Blackwell Publishing Ltd.

European standards. 2013. Available from Internet: <http://www.en-standard.eu/csn-en-13816transportation-logistics-and-services-public-passengertransport-service-quality-definition-targeting-andmeasurement $>$.

Harrison, S.; Henderson, G.; Humphreys, E.; Smyth, A. 1998. Quality bus corridors and green routes: can they achieve a public perception of 'permanence' of bus services?. In Proceedings of the European Transport Conference, 225-236.

Matulin, M.; Mrvelj, Š.; Gold, H. 2010. Evaluating the public transport priority system - Identifying background data impact. In Proceedings of the 17th ITS World Congress.
Matulin, M.; Mrvelj, Š.; Jelušić, N. 2011. Two-level evaluation of public transport system performances, Promet - Traffic \& Transportation, Scientific Journal on Traffic and Transportation Research. DOI: http://dx.doi. org/10.7307/ptt.v23i5.151, 23(5): 329-411.

Prioni, P.; Hensher, D.A. 2000. Measuring service quality in scheduled bus services, Journal of Public Transportation, 2(3): 51-74.

Pullen, W.T. 1991. The measurement of the quality of local bus services with respect to the effects of bus deregulation in Scotland, Newcastle: University of Newcastle upon Tyne, Ph.D. thesis.

Sun, H.; Si, B.; Wu, J. 2008. Combined model for flow assignment and mode split in two-modes traffic network, Journal of Transportation Systems Engineering and Information Technology. DOI: http://dx.doi.org/10.1016/S15706672(08)60035-5, 8(4): 77-82.

Thompson, K.; Schofield, P. 2007. An investigation of the relationship between public transport performance and destination satisfaction, Journal of Transport Geography. DOI: http://dx.doi.org/10.1016/j.jtrangeo.2006.11.004, 15(2): 135-144.

Vedagiri, P.; Arasan, V. 2009. Estimating modal shift of car travelers to bus on introduction of bus priority system, Journal of Transport System Engineering and Information Technology. DOI: http://dx.doi.org/10.1016/ S1570-6672(08)60092-6, 9(6): 120-129.

\section{jitte 193}

